\title{
Direct Evidence for Lip-Lip Interactions in Multi-Walled Carbon Nanotubes
}

\author{
Chuanhong Jin $(\varangle)$, Kazu Suenaga $(\varangle)$, and Sumio lijima \\ Nanotube Research Center, National Institute of Advanced Industrial Science and Technology (AIST), Tsukuba 305-8565, Japan \\ Received: 30 August 2008/ Revised: 27 September 2008/ Accepted: 27 September 2008 \\ (C)Tsinghua Press and Springer-Verlag 2008. This article is published with open access at Springerlink.com
}

\begin{abstract}
The stability of open edged multi-walled carbon nanotubes has been investigated by using in situ high resolution transmission electron microscopy at elevated temperatures. Formation of inter-shell structures was experimentally observed for the first time and attributed to a robust interaction between adjacent concentric shells (so-called lip-lip interaction). The fluctuating behavior of the inter-shell structures suggests a mechanism by which the carbon atoms can pass in or out through the inter-shell edges during carbon nanotube growth or shrinkage processes.
\end{abstract}

\section{KEYWORDS}

Multi-walled carbon nanotubes, lip-lip interaction, in situ transmission electron microscopy

\section{Introduction}

Multi-walled carbon nanotubes (MWNTs), consisting of well spaced concentric shells, are a promising material as a host for the intercalation of guest atoms and/or molecules. Therefore, applications as energy storage materials such as in lithium batteries are strongly anticipated [1]. One of the key elements that can affect their performance is the stability of the open edge at the ends of nanotubes. Due to the presence of dangling bonds and their residual reactivity, these open edges may become unstable, especially at high temperatures, which would adversely affect the intercalation of chemical species.

The issue of open edges is also of particular importance in understanding the formation mechanism of MWNTs, especially for a catalyst-free process like an arc discharge [2]. One of the most debated issues is whether the end of these MWNTs is open or closed during the growth. In the case of openend growth, a critical problem is the spontaneous closure of the open edge, which could terminate the growth $[3]^{1}$. Several theoretical groups have independently proposed a growth model involving the so-called lip-lip interaction between the open edges of neighboring concentric shells on an MWNT [5-8], which can effectively stabilize the open edges and facilitate the nanotube growth. To date, no direct experimental evidence for the formation of the liplip network has been obtained due to the difficulty of monitoring the rapid growth during the arc discharge process. Furthermore, in the limited theoretical reports, arguments still stand on whether the lip-lip network facilitates the open-end growth or vice versa.

Address correspondence to C. H. Jin, chuanhong-jin@aist.go.jp; K. Suenaga, suenaga-kazu@aist.go.jp

${ }^{1}$ Also, we have demonstrated that single-walled carbon nanotubes prefer to grow with a closed end in a special case-inner growth [4]. 
For example, Nardelli et al. found that, due to the atom diffusion between two shells through the lip-lip network, the nanotube would finally become capped, and the growth should then terminate [8]. Therefore, a clear and direct experimental study of the open edges of MWNTs at high temperatures is obviously important. In this work, we employed an in situ highresolution transmission electron microscopy (HRTEM) method to observe the evolution of the open edges and to evidence the formation of lip-lip networks on an MWNT.

\section{Experimental}

The experiments were carried inside a TEM (JEOL$2010 \mathrm{~F}$, operated at $120 \mathrm{kV}$ ) using a dedicated sample holder (Nanofactory), where a piezo-stage can drive a sharp tungsten (W) tip in three dimensions and manipulate individual MWNTs (made by arc discharge), as shown schematically in Fig. 1. We were able to apply a biased voltage between the $\mathrm{W}$ tip and the opposite palladium (Pd) electrode, and induce a current through a selected nanotube. Open edges were fabricated in situ by manipulating the starting MWNTs through an electrical breakdown

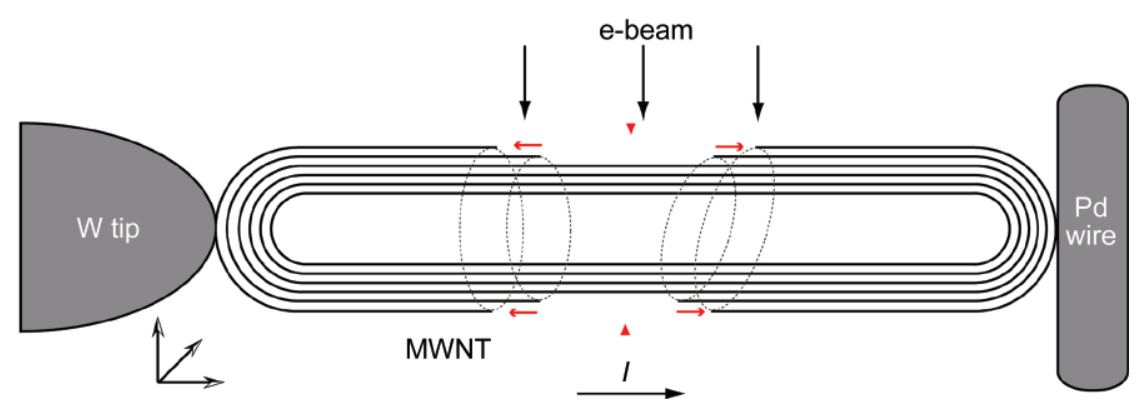

Figure 1 Schematic image of the experimental setup. A single MWNT is bridged by a Pd wire and a W tip. After applying a high biased voltage, open edges were formed on the outermost shell through electrical breakdown. These open edges then migrated toward both ends through thermally activated carbon evaporation. The electromigration and energetic electron irradiation further assist this process. Due to the temperature distribution gradient along the nanotube, the middle area is relatively hotter and therefore the carbon evaporation is slowed down and even terminated on the open edge. In this case, when we further increase the applied voltage, the second outermost shell breaks and due to its smaller diameter, carbon evaporation usually proceeds faster at this point. As a consequence, the open edges on the second outermost shell can catch up with their outer neighbors process with careful control of the induced current (Fig. 1) [9-11]. Images were digitally recorded by a slow scan CCD (Gatan-894) with an exposure time of $0.5-1.0 \mathrm{~s}$. The typical electron dose for each TEM frame was about $4 \times 10^{4}$ electrons $/ \mathrm{nm}^{2}$.

\section{Results and discussion}

In the first part, we will discuss the structural instability when only one open edge is formed at the outermost shell of the MWNTs. The most frequently observed phenomenon was the formation of active inter-shell defects (Fig. 2) as also reported by Huang et al. $[12,13]$. Due to the dangling bonds of the twocoordinate carbon atoms at the open edges they are expected to be highly reactive, especially at high temperatures. After an open edge was fabricated (indicated with blue arrows in Fig. 2(a)), the outermost shell joined partially with its nearest neighbor to form an inter-shell defect (indicated with a red arrow in Fig. 2(b)). Subsequently, a kink appeared which should correspond to the formation of a topological defect such as a pentagon-heptagon pair, since a topological defect is definitely required in order to seamlessly connect two nanotubes with different diameters and chiralities $[14]^{1}$. The whole structure then suddenly recovered just a few seconds later (Fig. 2(c)). After this open edge moved downwards about $3.8 \mathrm{~nm}$ through further carbon evaporation (Fig. 2(d)), the same type of inter-shell defect again formed on the nanotube. This time a partial joint first occurred on its left side (Fig. 2(e)) and a kink appeared again across the joint area (indicated with a red arrow). Note that the applied voltage and current (about $1.7 \mathrm{~V}$ and $84 \mu \mathrm{A}$, respectively) were kept unchanged throughout the experiment. At a certain temperature ${ }^{2}$, such a

\footnotetext{
${ }^{1}$ It is believed that there is no chiral angle correlation between two adjacent graphene shells in concentric MWNTs. For example Ref. [15].

${ }^{2}$ Here the temperature was estimated to be about 1500-2000 K. Similar or even higher temperatures were reported previously for the similar system in Ref. [9].
} 


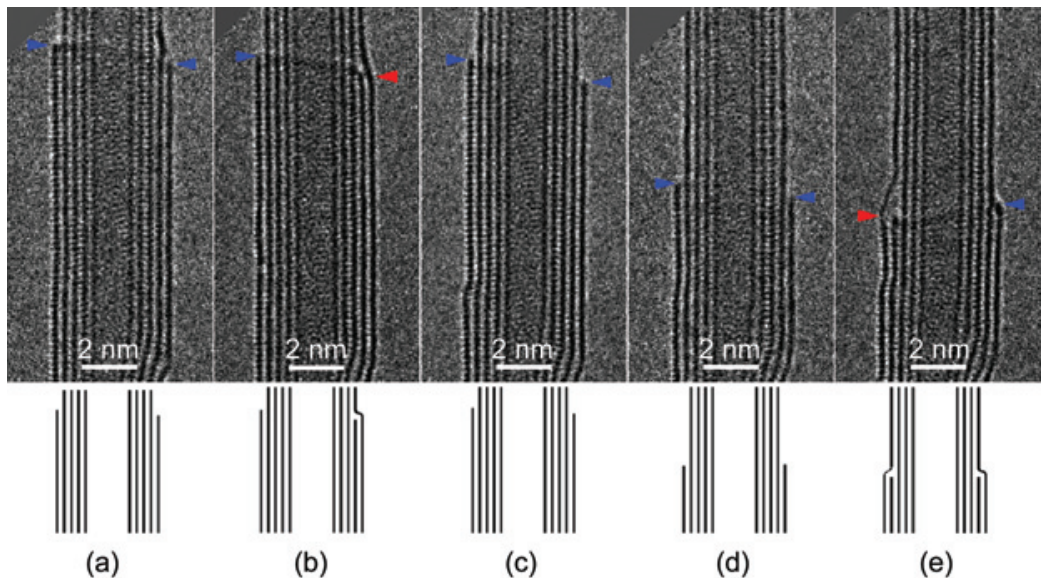

(a)

(b)

(c)

(d)

Figure 2 Formation and migration of active inter-shell defects: (a) an open edge (indicated with blue arrows) was fabricated on the outermost shell (The upper part is not shown); (b) when the outermost shell partially joined with the nearby shell a kink was formed across the joint area (see the red arrow). The other part remained unchanged; (c) the whole structure recovered; (d) the open edge moved downward by $3.8 \mathrm{~nm}$ through carbon evaporation at a high temperature; (e) this open edge again joined partially with its nearest neighbor, and a kink was formed across the joint area (see red arrow). During the whole process, the applied voltage and current were kept constant (at about $1.7 \mathrm{~V}$ and $84 \mu \mathrm{A}$, respectively)

transformation was repeatedly observed and the edge structure never became stabilized in so far as the open edge survives until the open edge becomes eventually closed.

Another important structural evolution of an MWNT with open edges is given in Fig. 3 as a series of HRTEM images. During the peeling process (Figs. 3(a) and 3(b)), the shrinking outermost shell suddenly joined to the neighboring (second) shell, and the remaining part of the second shell subsequently connected to the innermost shell at an applied (e)

voltage of $1.7 \mathrm{~V}$ with a current of $54 \mu \mathrm{A}$ (Figs. 3(a) and 3(b)). As a consequence, the innermost shell was thus isolated and then closed its cap immediately. Again there was a kink formed on each of the outer two shells across the joint area. In contrast to the case shown in Fig. 2, all the edges of the outer shells joined to their next neighbors consecutively. There were no remaining open edges and therefore the whole structure became stabilized and never reactivated, even when we further increased the applied voltage and current (Fig. 2(e)).

From the results shown above, we can conclude that an edge of an MWNT is quite unstable if only one shell is open edged. Then what would happen if we had a pair of open edges on the same nanotube? In the following part, we will focus on the interactions between the adjacent open edges and the formation of the lip-lip network. The serial HRTEM images shown in Fig. 4 give the first example. Two open edges were created at two outer shells (Fig. 4(a)) and then the upper one was driven to move down towards the other one through carbon evaporation under an applied voltage of $1.9 \mathrm{~V}$ and a current of $118 \mu \mathrm{A}$ (Fig. 4(b)). A few seconds later, a new structure with enhanced image contrast appeared just after these two open edges met each other. This is the so-called lip-lip

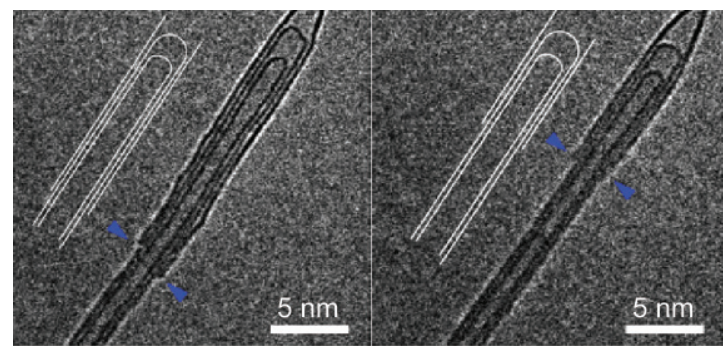

(a)

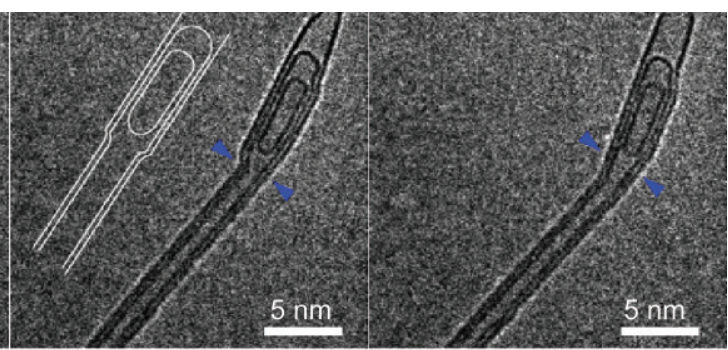

(c)

(d)

Figure 3 Formation, migration, and healing of another type of inter-shell defect. (a) An open edge was fabricated on the outermost shell (indicated with blue arrows). (b) Under a potential of $1.8 \mathrm{~V}$ with current of $54 \mu \mathrm{A}$, the open edge moved upwards by about $7.8 \mathrm{~nm}$ through carbon evaporation. (c) After a few seconds, the whole structure changed suddenly: the outermost shell seamlessly joined the bottom part of the middle one, and also the remaining part of the middle shell seamlessly joined with the bottom part of the innermost one. A closed cap was formed on the remaining part of the innermost shell and a kink formed on each outer shell across the joint area. (d) Once such a configuration was formed, it became very stable and never returned back to that shown in Fig. 2, even when the applied voltage was further increased 


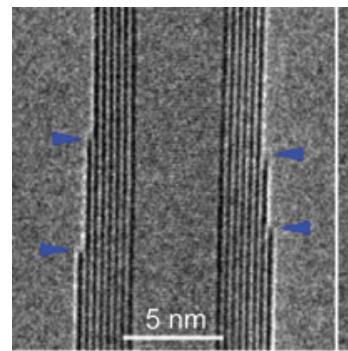

(a)

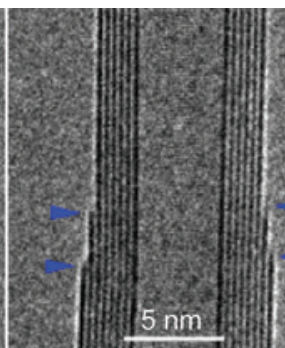

(b)

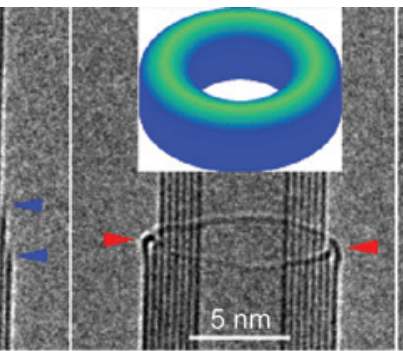

(c)

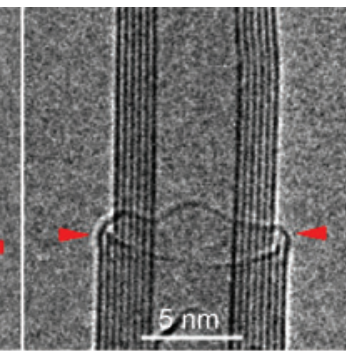

(d)

Figure 4 Serial HRTEM images showing the formation of a lip-lip network at high temperatures. (a) Two open edges (indicated with blue arrows) were fabricated on the MWNT, and (b) the upper open edge moved downwards. (c) A lip-lip network was formed between two open edges when they met each other. At the two sides (see red arrows) the local structures were found to match the curved graphitic shells. Inset is a schematic illustration of the lip-lip network. The applied voltage and current did not change during the whole process. (d) The lip-lip network underwent structure fluctuation, and sometimes a few protuberances were formed, especially when a higher biased voltage was applied

network, where chemical bonding occurred between the neighboring two-coordinate carbon atoms on the open edges of adjacent concentric shells (Fig. 4(c) and the inset) [5-8]. Closed or folded shells can be seen at both sides of the connected edges (indicated with red arrows), which is direct evidence for the formation of a lip-lip network. Most interestingly, after the lip -lip formation, the connected edges did not shrink further and no inter-shell defects were observed, even when the applied voltage was further increased. We did not observe any evidence for a separation of the two connected edges: once they meet, they never separate, which definitely means that the two open edges were energetically stabilized through the lip-lip formation.

It is quite reasonable that a few two-coordinate carbon atoms still exist on the lip-lip network because all the dangling bonds cannot be fully saturated, due to the intrinsic incommensurability of the graphene network as discussed from a theoretical viewpoint in Ref. [6]. Therefore, the liplip network should undergo a structural fluctuation involving a continuous reorganization of the bridging carbon bonds that are frequently breaking and reforming at high temperature. Such a structural fluctuation was indeed observed experimentally as shown in Fig. 4(d). A few local protuberances were occasionally found at the lip-lip network, indicating the fluctuating bond topology and associated carbon diffusion. This suggests that the inter-shell diffusion barrier for carbon atoms and clusters should be substantially decreased due to the formation of the lip-lip network. The smooth bumps formed at the edge are very specific to the edge structures observed at high temperatures.

We have tested over one hundred specimens and always observed a similar phenomenon. Another example is given in Fig. 5. It was found that such a lip-lip network could even be formed between three concentric open edges, as highlighted in Fig. 5(c). A

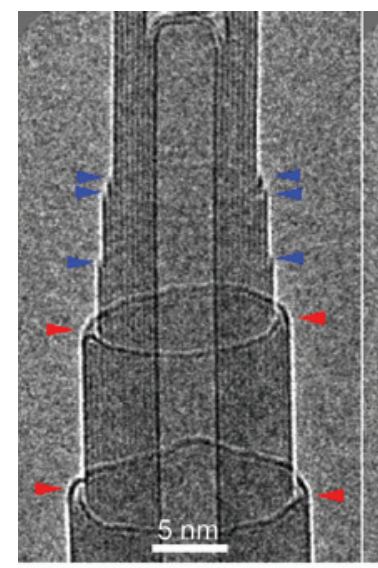

(a)

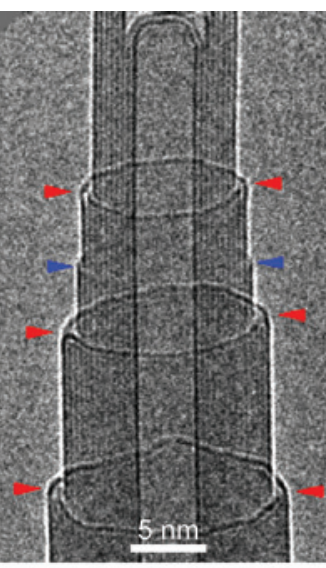

(b)

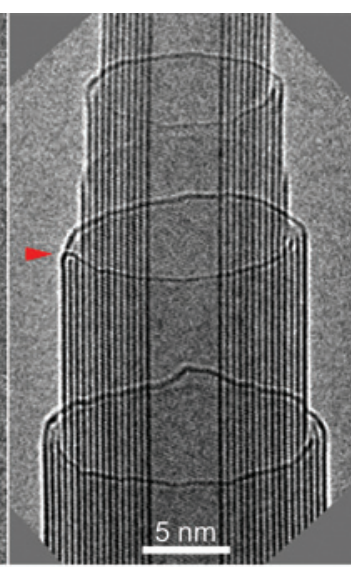

(c)

Figure 5 Another example showing the formation of lip-lip network: (a) two open edges were fabricated on an MWNT, and the upper one was driven to move downwards through carbon evaporation; (b) a lip-lip network was formed when these two upper open edges met; (c) an enlarged display of (b). Interestingly, the lower liplip network (indicated with red arrows) consisted of three open edges, and a sword-insheath like local structure was formed 
few sword-in-sheath like structures (indicated with red arrows) were found on these lip-lip networks, where the innermost and outmost edges form a link, with the middle one being shielded within this network.

Is it still possible to incorporate/ evaporate carbon atoms or clusters from the lip-lip network? Another very interesting issue is whether the lip-lip network can really act as the growth/shrinkage rim. This is of key importance for understanding

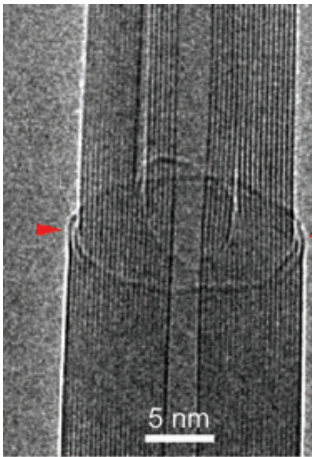

(a)

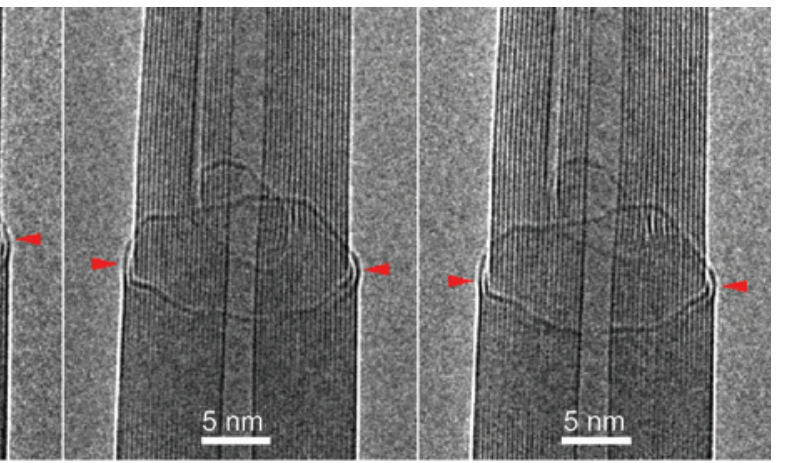

(b)

(c)
Figure 6 Consecutive HRTEM images showing the shrinkage of an as-formed lip-lip network by about $3.3 \mathrm{~nm}$ at high temperature. This means that the lip-lip network still remains active for the evaporation/incorporation of carbon atoms and clusters

shells might have prohibited the cap closure of the outer shells which theoretical predictions [8] suggest should have occurred in the absence of inner shells; (3) The actual temperature of the MWNT specimens in this experiment could not reach as high as $3000 \mathrm{~K}$, which is a typical temperature for the arc discharge synthesis and theoretical simulations $[3,6-7]$.

\section{Conclusions}

In summary, using in situ HRTEM, we presented here direct evidence for the formation of a lip-lip network between the open edges of the neighboring concentric shells of MWNTs, which can indeed stabilize the whole system, and might facilitate the growth of MWNTs with open ends in the absence of metal catalysts.

\section{Acknowledgements}

One of us (C. H. Jin) would like thank the Japan Society for Promotion of Science (JSPS) for a postdoctoral fellowship and Fang Lin for useful discussions. The work on microscopy is partly supported by CREST and KAKENHI (19054017).

\section{References}

[1] Fischer, J. E. Carbon nanotubes: A nanostructured material for energy storage. Chem. Innov. 2000, 30, 2127.

[2] lijima, S. Helical mirotubules of graphitic carbon. Nature 
1991, 354, 56-58.

[3] lijima, S.; Ajayan, P. M.; Ichihashi, T. Growth model for carbon nanotubes. Phys. Rev. Lett. 1992, 69, 31003103.

[4] Jin, C. H.; Suenaga, K.; lijima, S. How does a carbon nanotube grow? An in situ investigation on the cap evolution. ACS Nano 2008, 6, 1275-1279.

[5] Guo, T.; Nikolaev, P.; Rinzer, A. G.; Tomanek, D.; Bolbert, D. T.; Smalley, R. E. Self-assembly of tubular fullerenes. J. Phys. Chem. 1995, 99, 10694-10697.

[6] Charlier, J. C.; Vita, A. D.; Blase, X.; Car, R. Microscopic growth mechanisms for carbon nanotubes. Science 1997, 275, 647-649.

[7] Kwon, Y. K.; Lee, Y. H.; Kim, S. G.; Jund, P.; Tomanek, D.; Smalley, R. E. Morphology and stability of growing multiwall carbon nanotubes. Phys. Rev. Lett. 1997, 79, 2065-2068.

[8] Nardelli, M. B.; Brabec, C.; Maiti, A.; Rolan, C.; Bernholc, J. Lip-lip interactions and the growth of multiwalled carbon nanotubes. Phys. Rev. Lett. 1998, 80, 313-316.

[9] Collins, P. G.; Arnold, M. S.; Avouris, P. Engineering carbon nanotubes and nanotube circuits using electrical breakdown. Science 2001, 292, 706-709.

[10] Huang, J. Y.; Chen, S.; Jo, S. H.; Wang, Z.; Han, D. X.; Chen, G.; Dresselhaus, M. S.; Ren, Z. F. Atomic-scale imaging of wall-by-wall breakdown and concurrent transport measurements in multiwall carbon nanotubes. Phys. Rev. Lett. 2005, 94, 236802.

[11] Jin, C. H.; Suenaga, K.; lijima, S. Plumbing carbon nanotubes. Nat. Nanotechnol. 2008, 3, 17-21.

[12] Huang, J. Y.; Ding, F.; Yakobson, B. I. Dislocation dynamics in multiwalled carbon nanotubes at high temperature. Phys. Rev. Lett. 2008, 100, 035503.

[13] Huang, J. Y.; Ding, F.; Jiao, K.; Yakobson, B. I. Selftemplated growth of carbon-nanotube walls at high temperatures. Small 2007, 3, 1735-1739.

[14] lijima, S.; Ichihashi, T.; Ando, Y. Pentagons, heptagons and negative curvature in graphite microtubule growth. Nature 1992, 356, 776-778.

[15] Hashimoto, A.; Suenaga, K.; Urita, K.; Shimada, T.; Sugai, T.; Bandow, S.; Shinohara, H.; lijima, S. Atomic correlation between adjacent graphene layers in doublewall carbon nanotubes. Phys. Rev. Lett. 2005, 94, 045504. 